part of the aortic arch, in place of arising from the brachio-cephalic trunk as usual. When the right subclavian artery arises from the left part of the aortic arch, it directs its course to the right side, either by passing in front of the trachea, as Professor Blandin has observed, or behind it, or behind the cesophagus, to restore itself to its normal position. In these various circumstances, he (Professor Blandin) was curious to know what became of the nerve. Is it alike reflected on the artery, or did this anomaly of origin bring with it a modification in the nerve? This it was which 1 verified for the first time, in 1843 , in a preparation which may be seen in the museum of the school.

In this case the subclavian artery passed from left to right, and after having taken its origin from the left part of the arch of the aorta, passed between the cesophagus and the vertebral column, and thus reached the space between the scaleni. In this case, which has been recorded in Professor Cruveilhier's " Treatise on Anatomy," there was absence of the inferior laryngeal nerve. Since then, I have had occasion to examine another subject in which the same arterial peculiarity existed, and where there was alike absence of the recurrent nerve. In these two anomalies, this is the cliaracter of the pneumogastric:-it occupies the normal position; from its internal part are given off a great number of nervous filaments, which are distributed to the larynx, to the inferior part of the pharynx, to the trachea, and to the esophagus. All these nervous filaments, destined to replace the inferior laryngeal nerve, pass under the common carotid artery; some before this vessel, to distribute themselves to the parts which they are destined to innervate. These small nerves which supply the trachea and cesophagus with nervous energy, are on tbe whole alike in volume to those which normally furnish the recurrent. As for their length and direction, they are a little different, being longer and directed more transversely. The nerves which furnish usually the recurrent to the larynx, come, as in the case which occupies us, from the pneumogastric; this latter affords to the level of the larynx, a branch so voluminous, that it might be considered a veritable recurrent nerve; and which arrived at the inferior part of the larynx, is in every respect disposed of in the came way as the termination of inferior laryngeal nerve, a termination so well studied by Professor Blandin. The cardiac nerves furnished ordinarily by the recurrent, are given off by the pneumogastric.

Dr. Demarquay then observes, that in a functinnal point of view this anomaly is of no moment, justly remarking that in certain operations, as in tying the common carotid, it might occasion great embarrassment to the surgeon, and then concludes as follows:-

Such, then, are four cases where the abnormal origin of the right subelavian brings with it an important modification of the nervous vagus. Are these, then, accidental cases, or are they the sign of a phenomenon, always persistive when the subclavian (right) artery arises from the left part of the aortic arch; - and does this induce absence of the recurrent nerve? This I would not venture to say: further observations must prove it.-Prov. Med. \& Surg. Journ., Oct.18, from Gaz. Méd. de Paris, Sept. 9.

5. On the Chemical Phenomena manifested by different substances introduced into the Organism. By M. BERNARD.-The author has performed a very extensive series of experiments, of which the conclusions may be stated shortly as follows:

1. Certain combinations of metals, which take place readily out of the body, and even in the gastric secretions, are prevented from occurring in the blood, and other animal fluids, by the affinities of the metal for organic matter. This proposition was drawn from observations on the reactions of a salt of iron with prussiate of potash.

2. Certain chemical reactions of the nature of fermentation $(e, g$. amygdaline with emulsine, sugar with yeast), which commonly do not take place in the stomach, are easily developed in the blood.

3. When salts of certain acids, whose affinity for bases is feeble (as the hydrocyanic and the carbonic), are injected into the veins, they are decomposed, and the acid is liberated; the decomposition in these cases appearing to take place in the capillaries of the lung.

4. Certain salts (such as the prussiates of potash and the salts of iron) appear 
to pass in the stomach from a lower to a higher, and in the blood and urine from a higher to a lower, state of oxydation.

[T'Te extreme interest attaching to $M$. Bernard's individual experiments, induces us to give an analysis, in the present and the next Number, of such of them as appear to lead directly to the above results.]

First Series.-After a number of experiments, which were vitiated by the difficulty of procuring a salt of iron which could be injected in the veins of an animal without fatal consequence, $M$. Bernard discovered that the lactate of iron was quite innocuous when used in this way. He then caused a simultaneous injection of lactate of peroxide of iron, and of prussiate of potash, to be thrown iuto different veins in the same animal. The result was surprising. Instead of the blue colour, which might have been expected, tbe blood and the tissues of the body experienced no apparent change whatever; the only exception being the pyloric extremity of the stomach, where a vivid blne colour was developed. The experiment was repeated several times, with similar results. In one instance only the urine presented a dingy blue colour; in all the experiments, however, a few drops of strong sulphuric acid added to the urine produced a copious precipitate of Prussian blue. These results were not aflecled by the acid or alkaline charucter of the urine itself.

The conclusion from these experiments was, that the double decomposition here indicated could only take place in two sjtuations, viz., in the stomach, and occasionally to a slight extent in the urine. That the absence of the blue colour did not result from the insignificance of the quantities employed, was proved by another experiment. Into the veins of one rabbit the ordinary quantity of prussiate of potash in solution was thrown, and after some minutes the animal was bled. In another rabbit lactate of iron was injected, and bleeding likewise performed. On mingling the serum derived from these two rabbits, no reaction took plaoe; nevertheless the one serum was easily proved to contain iron, and the other prussiate of potash. On adding now to the mixed serun of the two animals a few drops of sulphuric acid, the blue precipitate was instantly produced. The action of the urine of the two rabbits when ningled, was precisely similar to that of the serum. It was different, however, with the gastrio fluid. On washing the coats of the stomach in the two animals, acid liquids were procured, which, on being mingled, gave rise to a blue colour immediately.

That this difference between the gastric and the other fluids was not due solely to acidity, was shown by the fact, that the urine presented the same phenomena, whether acid or alkaline, as above stated.

Having determined these facts, M. Bernard next proceeded to try the effect of tbe direct addition of the salt of iron, and afterwards of the prussiate of potash, to serum, urine, and gastric juice. In the first two he found that, except in the case where an acid was added, or large quantities of the re-agents used, there was no blue precipitate, while in the last the blue colour always appeared. When, however, the precipitate or potash was added first to the fluids, and aflerwards the iron salt, the blue colour was immediately produced in the whole.

M. Bernard accordingly concluded, that from the attraction of iron for animal substances, it is prevented from developing its usual relations with prussiate of potash, when both these substances are injected into the blood; but that if a strong acid be added to animal matter containing these salts, this affinity for the animal matter is destroyed, and the several chemical relations are established. This does not take place, however, on the addition of phosphoric, acetic, or lactic acids.

The peculiarity of the gastric juice in these experiments is explained by the author, on the ground of the small quantity of organic matter in it being less than any other secretion in the body. [May not the existence of free muriatic acid be a more valid explanation?]

The tendency of iron to combine with the tissues, is further illustrated by $M$. Bernard in three experiments. In the first, a solution of lactate of iron was introduced into the cellular tissue of the neck, and a similar quantity of solution of prussiate of potash into the right thigh. A blue colour soon became developed in the former situation, while the thigh retained its natural colour. In the second experiment, the salt of iron was introduced into the thigh, and prussiate of potash was injected into the veins; the blue colour became developed in the thigh. In 
the third experiment, the salt of potash was introduced into the cellular tissue, and the iron-salt into the veins. In this case tbere was no blue colour developed.

It is obvious from these experiments, that the prussiate of potash circulates rapidly in the general mass of the blood, even when introduced in to the system by a wound in the integuments; while the iron seizes on the tissue with which it is placed in contact, from which it is very sparingly absorbed into the circulation. Thus the mixture of the two salts is not effected, except at the point of introduction of the iron.

Second Series.-The author next proceeded to determine if tbe blood presented any obstacle to the establishment of certain catalytic reactions, which take place witb facility external to the organism. The action of emulsine on amygdaline is favourable for this purpose, inasmuch as a highly poisonous substance is instantly generated by their union. The experiments of M. Bernard satisfactorily determine, that the injection of these substances into the circulation, simultaneously, or at a short interval, causes death, with the usual symptoms of poisoning by prussic acid, and with the evolution of a strong smell in the breath of oil of bitter almonds. The injection of either of the substances separately, on the contrary, gave rise to no fatal results.

The action of diastase on starch in the blood might naturally be expected to be a good subject of experiment. But the serum of the blood has the singular property of producing the transformation of starch into sugar; hence experiments on this head were not instituted.

The transformation of sugar into alcohol, by means of yeast, was the next sibject of experiment. In two animals (a dog and a rabbit), cane sugar and yeast were simultaneously introduced in to the circulation in considerable quantity. Both animals died. 'The urine of the rabbit alone was examined; it remained quite free of sugar (which, under ordinary circumstances, passes, with great ease into the urine). The symptoms in both animals were those of a marked typhoid condition, with fever and extreme debility; the rabbit died in less than twenty-four hours, the dog on the third day. The morbid changes were, in both animals, injection, ecchymosis, ulceration of the stomach, ecchymotic patches in the small intestine, and a dark imperfectly coagulated state of the blood. In both cases (a sufficiently singular fact) the pancreas was diseased, the orifice of its ducts being tumid and red; its substance in the rabbit red and inflamed; in the dog, the seat of numerous small abscesses. These facts appear to indicate a special poisonous action, exercised by the alcoholic fermentation upon the animal organization; and also to prove that the change of sugar into alcohol may occur within the circulation.

Third Series.-The rapidly fatal effect of cyanuret of mercury, when introduced into the stomach, is readily explained by the action of the gastric juice, which, even externally to the body, decomposes it, setting free hydrocyanic acid. The action of this salt, when introduced into the veins, on the contrary, cannot be explained by the action of the blood upon it; as, out of the body, no decomposition takes place. Nevertheless, on injecting the cyanuret of potassium into the veins of an animal, a poisonous effect, similar to that of prussic acid, is produced; and the odour of prussic acid is exhaled from all the tissues.

Where, then, does this decomposition take place? As all the acid fluids of the body present a reaction similar to the gastric juice, $M$. Bernard performed the following experiment, to exclude the possibility of the decomposition taking place in the stomach or urinary passages. The stomach, kidneys, and bladdler, were removed from a live dog. A solution of cyanuret of mercury was then injected by the crural vein; in half a minuie the animal began to respire with difficulty, and died soon after, convulsed. All the tissnes were impregnated with the odour of prussic acid; yet in no place could an acid fluid be found. [Were the contents of the cacum examined? It appears to us that their well-known acidity forms an objection to tbis experiment.]

With the view of discovering whether this reaction took place in the systemic capillaries, the anthor now isolated the thigh of a dog from all communication with the rest of the body, leaving only the artery and vein entire. He then injectel by the artery a sufficient quantity of cyanuret of mercury, and shortly afterwards drew blood from the vein. The blood contained abundance of the salt, but no trace of prussic acid; the tissues of the thigh were in a similar condition. 
From this experiment, M. Bernard judges that the systemic circulation is not engaged in the decomposition of the cyanurets; he therefore, by way of exclusion, ascrihes this decomposition to the pulmonic circulation.

Certain bicarbonates are decomposed in the blood when passing the capillaries of the lung. Thus, if a large quantity of solution of bicarbonate of soda be injected into the veins of a rabbit, the animal dies suddenly, and, on dissection, there is found air in the division of the pulmonary artery, and sometimes even in the ventricles of tbe heart. The lungs also are emphysematous and distended. These results are not produced if the same salts are introduced gradually.

Fourth Series.-The experiments of this series are not so important as to require detailed notice; they consisted simply of an examination into the effects of different animal fluids, on the state of oxydation of the ferrocyanurets of potassium, and the salts of iron.-Monthly Journ. Med., \&c., July and Aug., 1848; froin Archives Générales de Médecine, Jan. and Feb., 1848.

\section{ORGANIC CHEMISTRY.}

6. On the Acidity and Allealinity of certain of the Human Fluids in the state of Health and Disease. By M. Andras.-In their physiological conditions, each of the humours of the body presents a certain degree of acidity or alkalinity; and the spontaneous transformation of a naturally acid fluid into an alkaline one, or vice versâ, never takes place in the healthy organism. The utmost that can occur in this respect, is the rendering the fluid temporarily neutral by great dilution, as in the case of excessive perspiration-1he water then being abstracted from the blood in larger proportion than the other principles. However this may be in health, the opinion is very generally entertained that in disease such chemical change in the humours does often take place; and the object of this paper is to investigate its accuracy.

Of all the fluids of the economy, the serum of the blood is the most decidedly alkaline; and whatever the nature of the disease or its duration, in wbich $M$. Andral has examined this fluid, he has never found the intensity of this reaction sensibly vary. Vogel quotes a case of metro peritonitis from Scherer, in which the serum of the blood is said to be perfectly neutral, but adds, that he himself had never met with anything similar. If blood is examined after death, any acidity then found is the result of decomposition, and not the effect of disease. In examining the condition of fluids formed from the blood, it should be borne in mind that upon the eame surfaces liquids possessed of different reactions may be found; so that the accidental predominance of one of these fluids may easily be mistaken for a change in the reaction of another. Thus the sweat is acid, but the sebaceous matter is allcaline. In the very varions conditions in health and disease under which M. Andral has examined the sweat, he has found it generally acid, sometimes from dilution neutral, never alkaline; but at the same time, at some parts of the skin, where sebaceous follicles abound, as the axilla and other hairy parts, an alkaline reaction may exist. It is evident, then, that the sweat is not a simple escape of the serum of the blool, charged with certain of its principles, for then it would be alkalime; and if the skin be irritated by blisters and the like, the fluid consequently effused will be found decidedly alkaline. So is the fluid fourd in herpes, eczema, pemphigus, \&c., vesicular diseases preceded by more or less congestion of the skin; and it is remarkable that the contents of sudamina, which unlike these are preceded by no congestion, are acid, being also destitute of albumen, which is found in the others. Although sudamina are usually accompanied by excessive sweating, cases of typhoid fever are sometimes met with where this is not the case.

Still more remarkable is the difference of the reactions of the various fluids found on mucous membranes, giving rise to considerable chance of error. Throughout their whole extent, they furnish, like the skin, an acid principle, which exists in the transparent fluid, destitute of globules, which they normally separate from the blood; but when this fluid is replaced by one of an opaque appearance and containing globules, secreted under the influence of acute or chronic inflainma- 